\title{
Educación a distancia en tiempos de COVID-19: Análisis desde la perspectiva de los estudiantes universitarios
}

\section{(Distance Education in COVID-19's period: An Analysis from the perspective of university students)}

\author{
Eva Pérez-López \\ Alfonso Vázquez Atochero \\ Santiago Cambero Rivero \\ Universidad de Extremadura, UNEX (España)
}

DOI: http://dx.doi.org/10.5944/ried.24.1.27855

\section{Cómo referenciar este artículo:}

Pérez-López, E., Vázquez Atochero, A., y Cambero Rivero, S. (2021). Educación a distancia en tiempos de COVID-19: Análisis desde la perspectiva de los estudiantes universitarios. RIED. Revista Iberoamericana de Educación a Distancia, 24(1), pp. 331-35o. doi: http://dx.doi.org/10.5944/ried.24.1.27855

\section{Resumen}

Este artículo sitúa el foco de interés en los estudiantes de las universidades presenciales y en los modelos de enseñanza a distancia (ED) implementados durante el periodo de confinamiento causado por la crisis del COVID-19. Los objetivos son analizar la incidencia del contexto personal y familiar en la equidad digital, identificar el modelo de enseñanza recibido, y conocer su valoración y percepción sobre este modelo. La investigación obedece a un estudio mixto de alcance descriptivo en el que se combinan métodos cualitativos y cuantitativos. En primer lugar, se realizó un cuestionario a estudiantes de la Universidad de Extremadura (UEx) $(\mathrm{n}=548)$ y a posteriori se efectuaron entrevistas online a miembros del equipo de gobierno de la universidad. Los resultados indican que los estudiantes procedentes de familias con un nivel formativo bajo tienen menos oportunidades de uso de tecnologías digitales. Las clases virtuales que han recibido han consistido esencialmente en presentaciones subidas al campus virtual con interacciones asíncronas. La valoración negativa que hacen de la enseñanza a distancia se explica por la inversa relación percibida entre dedicación al estudio y rendimiento académico y por falta de adaptación de los docentes a las circunstancias personales y académicas de los estudiantes. En conclusión, se determina que la universidad debe transitar hacia modelos más colaborativos y centrados en el estudiante.

Palabras clave: COVID-19, enseñanza superior; educación a distancia; equidad digital; estudiantes universitarios. 


\begin{abstract}
This paper focuses on the students of traditional face-to-face universities and on the implemented distance learning models during the lockdown period caused by the COVID-19' crisis. We aim here to analyze the impact of the personal and family context on digital equity, to identify the teaching model received, and to know their assessment and perception of this model. The research is a mixed study of descriptive scope in which qualitative and quantitative methods are combined. Firstly, a survey was carried out with students from the University of Extremadura $(n=548)$ and, then, online interviews were conducted to members of the university governance. The results indicate that students from families with a low educational level have fewer opportunities to use digital technologies. Virtual lessons, which students have received, have essentially been based on presentations uploaded to the virtual campus with asynchronous interactions. The negative assessment of distance learning is explained by the apparent reverse relationship between time spent studying and academic performance and by the lack of teachers' adaptation to students' personal and academic circumstances. In conclusion, the university must move towards more collaborative and student-centered models.
\end{abstract}

Keywords: COVID-19; higher education; distance education; digital equity; university students.

A partir de la declaración de pandemia por COVID-19 establecida por la Organización Mundial de la Salud (OMS) el 11 de marzo de 2020, prácticamente todos los países del mundo comenzaron a aplicar planes de preparación y respuesta a fin de tratar de controlar los efectos de este «ejército invisible», al decir de Harari (2016), en la población. Atendiendo a las recomendaciones de la OMS, el Gobierno de España decretó la limitación de la movilidad de las personas y la separación física en el ámbito social y laboral.

En tales condiciones, las universidades se vieron abocadas a la suspensión de los procesos de enseñanza-aprendizaje en su modalidad presencial y su continuidad en formato virtual. Así, además de los retos que venía enfrentando la universidad, esta precipitada transición la ha sometido a una prueba de estrés evidenciando uno de sus déficits estructurales, la equidad tanto en el acceso como en el progreso de la trayectoria académica (Ariño et al., 2019).

Las universidades presenciales tuvieron que migrar de forma urgente a lo que Hodges et al. (2020) han dado en llamar «enseñanza remota de emergencia». Muchas dosis de voluntarismo por parte de los docentes convirtieron los espacios de sus hogares en improvisadas aulas, al tiempo que, obligados por las circunstancias, se adentraron en el entorno de las tecnologías educativas. En la otra cara de la moneda se encontraban los estudiantes que sumaron a la incertidumbre propia de la extraordinaria situación, la de tener que enfrentarse y adaptarse a una modalidad telemática que exigía de ellos mayor compromiso y disciplina (UNESCO, 2020). 
Aunque es prematuro aventurar las secuelas educativas que a medio y largo plazo puede haber provocado el coronavirus, no lo es tanto señalar el agravamiento de las desigualdades en el caso de estudiantes cuyas familias poseen un escaso capital económico o cultural, como ponen de manifiesto diversos Organismos Internacionales (Banco Mundial, 2020; Organización para la Cooperación y el Desarrollo Económicos [OCDE], 2020; Organización de Estados Iberoamericanos [OEI], 2020; UNESCO, 2020; Fondo de las Naciones Unidas para la Infancia [UNICEF], 2020). En definitiva, los colectivos más damnificados por esta pandemia están siendo los más alejados de la cultura escolar, que pasa a ser también una cultura digital, «la cultura escolar de la era digital» (Beltrán Llavador et al., 2020, p. 94).

Si bien esta crisis está dejando a su paso un reguero de interrogantes en relación con el futuro de la educación, muchos de ellos seculares, no es menos cierto que nadie duda de que ésta va a cambiar, otra cosa será en qué dirección lo haga. Tome el rumbo que tome, es imprescindible analizar la calidad de la enseñanza remota recibida y las dificultades personales y académicas que han enfrentado los estudiantes a fin de disponer de diagnósticos basados en evidencias que permitan a las autoridades universitarias diseñar escenarios educativos en el corto y medio plazo.

\section{MARCO TEÓRICO}

Partimos de la premisa, como afirman Zubillaga y Gortazar (2020), de que la educación a distancia implica la planificación y el diseño de experiencias de enseñanza y aprendizaje online. Sin embargo, la celeridad con la que las instituciones de educación superior tuvieron que adoptar la medida del cierre de las aulas no dejó margen de maniobra para realizar estas tareas, de ahí el concepto de enseñanza remota de emergencia. Siguiendo las recomendaciones de la Red Española de Agencias de Calidad Universitaria (REACU, 2020) se adopta la terminología Educación o Enseñanza 'A Distancia'; no obstante, dado que en la literatura científica se suelen manejar también los términos e-learning o enseñanza online, los emplearemos indistintamente a lo largo del texto.

La ED posee una naturaleza y alcance complejos al implicar una amplia gama de formas no tradicionales de enseñanza y aprendizaje. En líneas generales, es una enseñanza que ocurre lejos del lugar del aprendizaje, requiere el uso de tecnologías (Moore y Kearsley, 2012), permite una gestión flexible del tiempo y otorga mayor autonomía al alumnado (Vlachopoulos y Makri, 2019).

Existen ya evidencias que señalan que el cierre de centros educativos causado por el COVID-19 ha agrandado la desigualdad de oportunidades, de manera singular en aquellas familias que poseen un capital sociocultural y económico bajo (Cabrera, 2020; Cabrera, Pérez y Santana, 2020). Los factores que actúan como agravantes de la desigualdad socioeducativa son los relacionados con la tipología de hogares de los estudiantes (composición familiar, número de hijos, niveles educativos de las madres y/o los padres) y los recursos de los que disponen para el seguimiento de la ED. 
En este sentido, Cabrera (2020) constata, para el caso español, que el alumnado de familias desfavorecidas, de centros públicos y del sur (incluyendo Galicia y Canarias) han sido especialmente afectados por la suspensión de las clases presenciales. Añade, además, que los desiguales niveles de renta, los diferentes niveles de estudio de las familias y las tipologías del hogar son determinantes en el acceso a un modelo educativo virtual telemático.

Para evitar la desigualdad digital, no solo es importante disponer de equipamiento tecnológico y conectividad sino el tipo de ordenadores que poseen los estudiantes - uso propio o compartido con otros familiares o con otros estudiantesy el tipo de conectividad -fibra óptica, ADSL, etc. - (Fernández Enguita, 2016). Pese a los esfuerzos institucionales, al menos en lo que a acceso a las Tecnologías de la Información y la Comunicación (TIC) se refiere, las inequidades en las aulas universitarias persisten (Alva, 2014) y, con ellas, diferentes perfiles tecnológicos. Toda esta amalgama de realidades genera un ecosistema "plurimodaltic» (SevillanoGarcía et al., 2016) que impacta de forma directa en la igualdad de oportunidades educativas.

A efectos de esta investigación consideramos dos elementos claves en la ED, en primer lugar, la interacción (síncrona o asíncrona) entre estudiantes y docentes; y, en segundo lugar, los recursos pedagógicos utilizados por estos últimos en las clases remotas.

Como ponen de manifiesto distintos estudios, uno de los elementos centrales de la educación online es la interacción entre el estudiante y el docente (Francescucci y Rohani, 2018; Hogg y Lomicky, 2012; Ragusa, 2017; Strang, 2013; Watts, 2016), en la que no solo es importante la cantidad (Castaño-Muñoz et al., 2014) sino también la calidad de la interacción (Brodie et al., 2013; Goldman, 2011). La evidencia empírica señala los beneficios del aprendizaje síncrono en el sentido de que proporciona más inmediatez que el asíncrono (Baker, 2011; Chakraborty y Nafukho, 2015; Francescucci y Rohani, 2018) y rompe la sensación de aislamiento al generar un sentimiento de pertenencia (Ragusa, 2017; Watts, 2016; Yang y Liu, 2007), además de mejorar el rendimiento (Duncan et al., 2012). Otros autores, sin embargo, respaldan en sus conclusiones las preferencias de los estudiantes por un modelo de interacción que combine modos síncronos y asíncronos porque optimizan la experiencia del aprendizaje (Falloon, 2011; Giesbers et al., 2013; Hrastinski, 2008; Moallen, 2015).

Gracias a la proliferación de plataformas tecnológicas de apoyo a la docencia, se ha ido asentando progresivamente un modelo blended-learning en la enseñanza universitaria (Llorente y Cabero, 2009) caracterizado por una combinación entre procesos de enseñanza-aprendizaje presenciales con otros virtuales (Fariña-Vargas et al., 2013) que permite generar situaciones de aprendizaje diversas.

A pesar de que las aulas virtuales admiten, a través de múltiples herramientas -presentaciones, vídeos, blogs, Webquest, foros de discusión, de novedades o de dudas, redes sociales, tareas, wikis, etc. - , la gestión de contenidos e información, la promoción de la comunicación y la colaboración, la realización de tutorías, además 
de la evaluación, su uso es poco frecuente en el desarrollo de las actividades docentes (Marcelo-García et al., 2015) y, en todo caso, se limita al uso básico de recursos para mantener el modelo pedagógico del profesor como transmisor de contenidos (Maor y Currie, 2017). De ahí que los correos electrónicos, las presentaciones visuales, los entornos o plataformas virtuales y las herramientas para intercambio de archivos sean las herramientas de mayor uso (Mercader y Gairín, 2017; Prendes, 2010), dejando un amplio abanico sin explorar (Mercader, 2019).

\section{METODOLOGÍA}

Esta investigación persigue conocer el modelo de enseñanza a distancia que han recibido los estudiantes de la UEx como consecuencia de la suspensión de las clases presenciales. A partir de la revisión de la escasa literatura existente sobre los impactos de la crisis sanitaria en el ámbito de la educación superior, consideramos la pertinencia de realizar un estudio que contribuya al diseño y planificación de decisiones en el ámbito universitario ante la incertidumbre que sigue generando la evolución del COVID-19. Así, se plantean los siguientes objetivos: 1) analizar la incidencia del contexto personal y familiar de los estudiantes en la equidad digital; 2) identificar el modelo de enseñanza a distancia implementado; y 3) conocer la percepción y valoración que hacen los estudiantes de este modelo.

\section{Características de la muestra}

La representatividad de la muestra para un nivel de confianza del 95\% y un margen de error del $5 \%$ requería un tamaño muestral $\mathrm{n}=377$; sin embargo, la investigación que se presenta tiene un tamaño muy superior $(\mathrm{n}=548)$. La población del estudio la forman 383 mujeres $(69,9 \%)$ y 165 hombres $(30,1 \%)$, con una media de edad de 22 años. El intervalo de edad 18-22 años es el que tiene mayor peso $(68,6 \%)$ coincidiendo con el rango etario promedio de 21,9 años. Las titulaciones de Grado en las que están matriculados los estudiantes se agrupan en cinco áreas de conocimiento y se añade la categoría genérica máster en la que se incluyen los estudiantes de Posgrado. El mayor porcentaje de estudiantes, 35\%, se concentra en el área de Ciencias Sociales y Jurídicas, seguido de un 32,3\% en el área de Ciencias de la Salud. De las 62 titulaciones de Grado que ofrece la UEx, prácticamente están todas representadas salvo cinco: Administración y Gestión Pública, Ciencias Ambientales, Podología, Psicología e Ingeniería Geométrica y Topografía.

\section{Instrumentos de recogida de datos}

Se realizó una investigación mixta de alcance descriptivo en la que se combinaron métodos cualitativos y cuantitativos. Los primeros consistieron en dos entrevistas, 
estructurada y semiestructurada, al vicerrector de Transformación Digital y a la vicerrectora de Estudiantes, respectivamente. En cuanto a los métodos cuantitativos, se diseñó un cuestionario exprofeso cuya validación se sometió al juicio de seis expertos que valoraron su univocidad y pertinencia. Las aportaciones de los expertos conllevaron el ajuste de algunos ítems. Para comprobar la consistencia interna se utilizó el Alfa de Cronbach que dio una puntuación de .815.

La encuesta se elaboró a través de la herramienta de Formularios de Google para permitir el envío masivo y una posterior retroalimentación online de los datos. Constaba de veintiséis preguntas estructuradas de las cuales catorce se referían a datos personales y sociodemográficos (edad, sexo, estado civil, curso en el que están matriculados, titulación en la que cursan estudios, etc.). Las restantes se agrupaban en cuatro dimensiones: 1) Datos contextuales, con el fin de conocer las circunstancias personales en la que los estudiantes estaban viviendo el confinamiento. Así, por ejemplo, se preguntaba por la situación laboral antes y después de decretarse el estado de alarma, por el domicilio de residencia, es decir, si se encontraban en un domicilio propio, familiar, compartido con otros estudiantes, segunda residencia u otros, y por el tamaño del municipio en el que vivían durante el confinamiento; 2) Disponibilidad de recursos tecnológicos para el seguimiento de las sesiones de enseñanza virtual: equipamiento informático, conectividad, calidad de la conexión, etc.; 3) Modelo de ED recibida: consistía en preguntas con escala tipo Likert sobre tipo de herramientas digitales usadas por los docentes y forma de interacción síncrona o asíncrona; y, 4) Percepción y valoración de los estudiantes sobre la docencia virtual, que agrupaba también preguntas con escala tipo Likert.

La recogida de datos se inició a finales de abril, habiendo transcurrido más de un mes desde la publicación de la resolución rectoral que dictaba la impartición de la docencia en régimen no presencial, y se prolongó hasta mediados del mes de mayo, después de que se diera a conocer a los estudiantes los nuevos criterios a aplicar en la evaluación final.

\section{ANÁLISIS Y RESULTADOS}

Los hallazgos que se presentan a continuación parten del análisis del cuestionario y las entrevistas. Éstas últimas permiten complementar algunos de los resultados obtenidos a través del cuestionario. Los datos estadísticos se analizaron con el programa SPSS versión 22.0.

\section{Incidencia del contexto personal y familiar de los estudiantes en la equidad educativa}

La mayoría de los estudiantes encuestados (92\%) están solteros y no tienen hijos (99\%), confirmando así el grupo etario juvenil. En cuanto a su situación laboral también el $92 \%$ se incluye en la categoría 'estudiante' y, por tanto, no dispone de 
empleo ni antes ni durante el confinamiento. Si antes de empezar la crisis sanitaria un $5 \%$ afirma tener empleo por cuenta ajena, durante el confinamiento este dato se reduce al 2,4\% y un $2 \%$ pasa a formar parte de un Expediente de Regulación Temporal de Empleo.

Teniendo en cuenta los datos anteriores, no es de extrañar que el $77 \%$ resida en el hogar familiar durante el confinamiento, frente a los que dicen hacerlo en el domicilio particular (9\%) o en una vivienda compartida (9\%). Atendiendo al tamaño del municipio de procedencia, los estudiantes se distribuyen fundamentalmente entre municipios de entre 1.000 y 5.000 habitantes (22\%) y localidades de más de 100.000 (21,8\%). En términos absolutos, el porcentaje de estudiantes que ha vivido el confinamiento en el entorno rural (54.6\%) es mayor que el que lo ha hecho en el urbano -localidades de más de 30.000 habitantes- $(45,4 \%)$.

El nivel formativo de los padres y/o madres oscila entre dos extremos: en uno se sitúan aquellos progenitores con estudios superiores (28\% de madres y/o $24 \%$ de padres) y, en el otro, los que carecen de estudios (2\% de madres y/o $5 \%$ de padres). Entre este rango están las madres y/o padres con Estudios Primarios (13\% madres y/o 17\% padres), con titulación en Educación Secundaría $1^{\text {a }}$ etapa (18\% madres y/o 15\% padres), en Educación Secundaría $2^{\mathrm{a}}$ etapa (18\% madres y $13 \%$ padres), y en Formación Profesional (16\% en ambos casos). Agrupando los porcentajes de estudiantes cuyos ascendentes están graduados en Secundaria $2^{\mathrm{a}}$ etapa, Formación Profesional o estudios universitarios, se obtiene que el 62\% de madres y 53\% de padres poseen un nivel de formación medio y alto, frente a las familias que carecen de estudios, o solo han alcanzado los Estudios Primarios o la Educación Secundaria $1^{\mathrm{a}}$ etapa, $35 \%$.

El tipo de equipamiento tecnológico con el que los estudiantes realizan el seguimiento de la ED es, por este orden: ordenador portátil propio $(73,2 \%)$; ordenador portátil compartido (16,8\%); ordenador de escritorio propio $(4,2 \%)$; smartphone personal $(2,2 \%)$; ordenador de escritorio compartido $(2,0 \%)$, tableta propia $(1,5 \%)$ y tableta compartida $(0,2 \%)$. Frente al $79 \%$ de los estudiantes que pueden hacer un uso individual de su equipamiento tecnológico, existe un $21 \%$ que, o bien lo comparte con otros miembros de la unidad familiar (19\%) o bien las condiciones de acceso están limitadas a las prestaciones que les ofrece la pequeña pantalla de su teléfono móvil (2\%). El cruce de variables relativas al equipamiento tecnológico y el nivel educativo de las familias (tabla 1) reporta que poseen portátil propio el $17,5 \%$ de los estudiantes cuyos padres son titulados superiores y el $19 \%$ cuyas madres tienen esa misma graduación universitaria. Este dato contrasta con el de aquellos estudiantes con idéntico equipamiento y cuyos ascendentes carecen de estudios, solo un 3,6\% de estudiantes, en el caso de padres sin estudios, y un 1,1\%, en el de madres sin estudios. 


\section{E. Pérez López; A. Vázquez Atochero; S. CAmbero Rivero}

EduCACIÓN A DISTANCIA EN TIEMPOS DE COVID-19: ANÁLISIS DESDE LA PERSPECTIVA DE LOS ESTUDIANTES UNIVERSTTARIOS

\begin{tabular}{|c|c|c|c|c|c|c|c|c|c|}
\hline 을 & 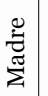 & -1 & -1 & $\underset{\sim}{N}$ & $\infty$ & 0 & 0 & -1 & $\stackrel{\infty}{\infty}$ \\
\hline 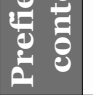 & 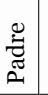 & N & N & $\stackrel{\leftrightarrow}{N}$ & $\Lambda$ & 0 & 0 & N & $\begin{array}{l}\infty \\
\infty\end{array}$ \\
\hline 8 & 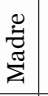 & 0 & $\infty$ & $\Lambda$ & $\nabla$ & 0 & 0 & - & $\stackrel{10}{\sim}$ \\
\hline ठே & 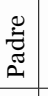 & 0 & $\nabla$ & $\stackrel{-}{\circ}$ & $\forall$ & -1 & 0 & -1 & 으 \\
\hline$\Xi \stackrel{0}{\frac{0}{\sigma}}$ & 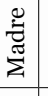 & -1 & $N$ & 0 & -1 & 0 & 0 & 0 & $\stackrel{ }{-1}$ \\
\hline c $\frac{\overrightarrow{0}}{8}$ & 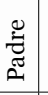 & $r$ & 0 & શે & 10 & 0 & 0 & 0 & ㄴ. \\
\hline ฮ็ & 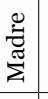 & $\infty$ & -1 & $\stackrel{\infty}{+}$ & $\stackrel{1}{\sim}$ & 0 & 0 & $\nabla$ & $\vec{\lambda}$ \\
\hline$E$ & 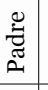 & N & N & $\hat{\sigma}$ & $\underset{\sim}{ }$ & 0 & 0 & 10 & ฟ \\
\hline$\frac{\pi}{\frac{\pi}{7}} \frac{\pi}{5}$ & 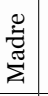 & $-r$ & 0 & $\infty$ & $\stackrel{0}{-1}$ & 0 & 0 & 0 & ฉ \\
\hline हैं : & 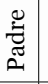 & 0 & 0 & ชิ & $\bar{F}$ & 0 & 0 & $\neg$ & $\vec{\infty}$ \\
\hline 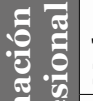 & 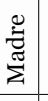 & $\infty$ & 0 & $\bar{\Sigma}$ & 0 & N & 0 & 0 & $\infty$ \\
\hline $\begin{array}{ll}0 \\
0 \\
0\end{array}$ & 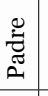 & $a$ & 0 & in & $\mathbb{J}$ & $N$ & 0 & $N$ & 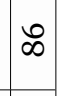 \\
\hline$\frac{\frac{\pi}{2}}{\frac{\pi}{5}} \frac{\pi}{5}$ & 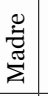 & 0 & -1 & ス & 9 & $\infty$ & -1 & N & ลิ \\
\hline ల్ల & 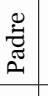 & N & -1 & 10 & 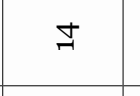 & $\neg$ & -1 & 0 & 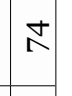 \\
\hline 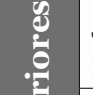 & 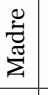 & $a$ & $m$ & ণ্- & $\stackrel{\infty}{N}$ & $\infty$ & 0 & $\nabla$ & $\overrightarrow{5}$ \\
\hline$\frac{8}{3}$ & 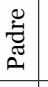 & $\Lambda$ & N & ฉू & $\overrightarrow{\mathrm{N}}$ & $\nabla$ & 0 & $\neg$ & $\vec{n}$ \\
\hline & & 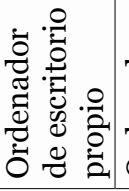 & 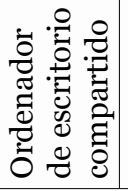 & 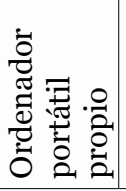 & 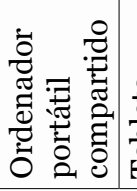 & 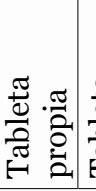 & 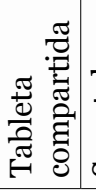 & 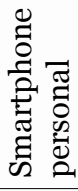 & 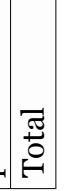 \\
\hline
\end{tabular}


En cuanto al acceso a Internet (tabla 2), el $54,2 \%$ se conecta mediante fibra óptica propia; el $24 \%$ a través de ADSL propia; un 6,6\% lo hace con fibra óptica compartida; un 6,2\% utiliza los datos de alta velocidad de su móvil; un 3,6\% también accede a través de los datos (baja velocidad) de su móvil; el 3,5\% cuenta con ADSL compartida; un 1,6\% utiliza otras formas de conexión; y, finalmente un $0,4 \%$ no posee ningún tipo de conectividad. Estos datos reflejan la misma tendencia que muestra la tabla 1; esto es, el porcentaje de los que disponen de conectividad propia $(78,2 \%)$ respecto a los que, o bien comparten la conectividad (10,1\%), o utilizan los datos de su móvil $(9,8 \%)$ o carecen de ella $(0,4 \%)$.

$\mathrm{Al}$ analizar los datos de conectividad en función del nivel de estudios de las familias, se vuelve a corroborar que en aquellos hogares donde alguno de los miembros de la unidad familiar posee estudios superiores, la disponibilidad de fibra óptica propia (16\%) está por encima de los hogares en los que alguno de los miembros o los dos no tienen estudios $(1,4 \%)$. Lo mismo puede decirse en relación con aquellos estudiantes que disfrutan de ADSL propia: si el 4,4\% de los que tienen este tipo de conexión son hijos de familias con estudios superiores, el 0,7\% procede de familias sin estudios. 


\begin{tabular}{|c|c|c|c|c|c|c|c|c|c|c|}
\hline & & ลิ & : & $\vec{\oplus}$ & $\stackrel{9}{=}$ & m & હิ & $a$ & N & $\begin{array}{l}\infty \\
\dot{1} \\
10\end{array}$ \\
\hline $\begin{array}{ll}0 \\
0 \\
0 \\
0 \\
0\end{array}$ & 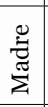 & $a$ & $\neg$ & $\nabla$ & 0 & N & $N$ & 0 & 0 & $\underset{\sim}{\infty}$ \\
\hline 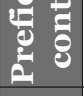 & 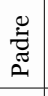 & ลี & $\infty$ & $\Lambda$ & $r$ & N & $N$ & -1 & 0 & 冓 \\
\hline 8 & $\begin{array}{l}\stackrel{0}{\overparen{t}} \\
\sum \\
\Sigma\end{array}$ & 0 & N & N & $r$ & $r$ & $\infty$ & 0 & 0 & $\stackrel{10}{\sim}$ \\
\hline 8 & 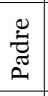 & $\exists$ & 0 & $\infty$ & 0 & $\infty$ & $\infty$ & 0 & 0 & คి \\
\hline$=\frac{8}{8}$ & 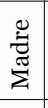 & $\infty$ & N & N & $r$ & 0 & 0 & $N$ & 0 & $\stackrel{ }{-1}$ \\
\hline (5) & 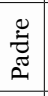 & $\stackrel{N}{\sim}$ & N & 0 & N & 0 & 0 & $m$ & - & $\stackrel{\text { ֻ }}{ }$ \\
\hline 苞 & 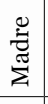 & $\vec{\nabla}$ & $\nabla$ & $\stackrel{10}{\sim}$ & $r$ & $\infty$ & 10 & N & 0 & 지 \\
\hline E & 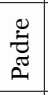 & $\stackrel{\infty}{m}$ & 10 & ๗ి & $\infty$ & 10 & 0 & $\infty$ & 0 & ฟ \\
\hline 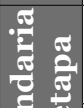 & 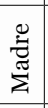 & $\stackrel{q}{q}$ & $r$ & $\hat{n}$ & 10 & 0 & $N$ & $N$ & 0 & ন \\
\hline 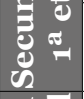 & 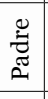 & ફे & $\infty$ & $\stackrel{\text { ֻ }}{ }$ & 10 & $\nabla$ & $\infty$ & 0 & -1 & $\infty$ \\
\hline 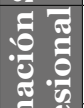 & 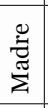 & นึ & $\Lambda$ & $\stackrel{\infty}{\infty}$ & $\nabla$ & $N$ & $N$ & -1 & 0 & $\infty$ \\
\hline $\begin{array}{ll}0 \\
0 \\
0 \\
0\end{array}$ & 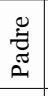 & กิ & 0 & ผึ & $n$ & $N$ & 0 & 0 & 0 & $\infty$ \\
\hline 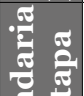 & 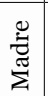 & 今 & $\exists$ & ๙ึ & $m$ & $a$ & $\nabla$ & 0 & 0 & ลิ \\
\hline 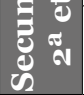 & 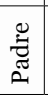 & ફે & 10 & $\stackrel{\sim}{\sim}$ & $r$ & $a$ & $\nabla$ & 0 & 0 & 六 \\
\hline$\stackrel{0}{0}$ & 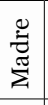 & ฟู & $\infty$ & ి्లి & $\nabla$ & $\Rightarrow$ & $N$ & N & N & 됴 \\
\hline हैं & 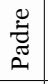 & চ & $\stackrel{N}{\sim}$ & $\stackrel{\infty}{\sim}$ & $\nabla$ & $a$ & $N$ & $\mathrm{~N}$ & 0 & $\vec{\oplus}$ \\
\hline & & 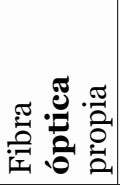 & 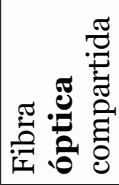 & 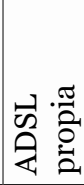 & 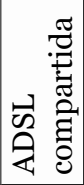 & 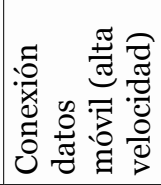 & 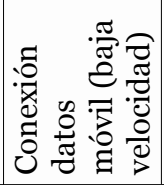 & 胥 & 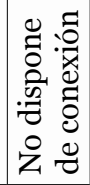 & 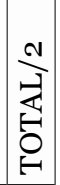 \\
\hline
\end{tabular}




\section{Modelo de enseñanza a distancia}

El cuestionario preguntaba a los estudiantes sobre el tipo de recursos utilizados por los docentes en la modalidad de ED e incorporaba en todos los ítems relacionados con este tema las formas de interacción profesorado-alumnado (síncrona o asíncrona), en caso de haberlas.

Los datos (tabla 3) proyectan que 'todos' o 'la mayoría' de docentes acuden preferentemente a las presentaciones para impartir ED, ya sea de forma asíncrona (44,3\%), síncrona (15\%) o sin ningún tipo de interacción (19\%). Sin embargo, el mayor porcentaje se concentra en aquellos estudiantes que consideran que 'algunos' docentes han utilizado videotutoriales para impartir docencia y han interaccionado con los alumnos a través del foro del campus virtual o el correo electrónico $(63,0 \%)$, seguido de los que afirman que 'algunos' han usado presentaciones $(61,5 \%)$ y videotutoriales $(61,1 \%)$, en ambos casos, comunicándose con los estudiantes mediante videoconferencias para la resolución de dudas. Finalmente, un 14,6\% asegura no haber recibido enseñanza online de 'ninguno' de sus profesores.

Tabla 3. Modalidad de educación a distancia e interacción con los estudiantes

\begin{tabular}{|l|c|c|c|c|c|c|c|}
\hline & $\begin{array}{c}\text { Presentación } \\
\text { e interacción } \\
\text { asíncrona }\end{array}$ & $\begin{array}{c}\text { Presentación } \\
\text { e interacción } \\
\text { síncrona }\end{array}$ & $\begin{array}{c}\text { Presentación } \\
\text { sin } \\
\text { interacción }\end{array}$ & $\begin{array}{c}\text { Videotutorial } \\
\text { e interacción } \\
\text { asíncrona }\end{array}$ & $\begin{array}{c}\text { Videotutorial } \\
\text { e interacción } \\
\text { síncrona }\end{array}$ & $\begin{array}{c}\text { Videotutorial } \\
\text { sin } \\
\text { interacción }\end{array}$ & $\begin{array}{c}\text { No se } \\
\text { imparte } \\
\text { docencia }\end{array}$ \\
\hline Todos & $12,2 \%$ & $4,2 \%$ & $2,9 \%$ & $2.2 \%$ & $2,2 \%$ & $0,4 \%$ & $7,1 \%$ \\
\hline Mayoría & $32,1 \%$ & $10,8 \%$ & $16,1 \%$ & $12,0 \%$ & $8,2 \%$ & $3,3 \%$ & $18,1 \%$ \\
\hline Algunos & $47,4 \%$ & $61,5 \%$ & $47,6 \%$ & $63,0 \%$ & $61,1 \%$ & $33,8 \%$ & $60,2 \%$ \\
\hline Ninguno & $8,2 \%$ & $23,5 \%$ & $33,4 \%$ & $22,8 \%$ & $28,5 \%$ & $62,6 \%$ & $14,6 \%$ \\
\hline TOTAL & $100 \%$ & $100 \%$ & $100 \%$ & $100 \%$ & $100 \%$ & $100 \%$ & $100 \%$ \\
\hline
\end{tabular}

Fuente: Elaboración propia

Las entrevistas parecen confirmar la traslación del modelo de enseñanza tradicional basado en clases magistrales al entorno virtual. De ahí que la utilización de presentaciones sea la principal herramienta a la que recurren los docentes.

Hay un porcentaje de profesorado con muy altas competencias [digitales] mientras que a otros les cuesta adaptarse a las circunstancias extraordinarias [...] En general, la comunidad universitaria no está preparada para la actividad académica en entornos virtuales; es necesario conocer más herramientas y recursos orientados a la docencia virtual. $Y$, sobre todo, hay que cambiar el tipo de enseñanza. No podemos sustituir una docencia de tres horas en clase presencial por tres horas de clase por videoconferencia [...] La creación de títulos universitarios virtuales va mucho más allá de poner a disposición de nuestros estudiantes contenidos PDF en el campus virtual (vicerrector de Transformación Digital). 
Una clase online no puede consistir en un profesor que hace lo mismo que en una clase presencial. El aprendizaje memorístico consistente en subir un PDF o un PowerPoint al Campus Virtual es un modelo obsoleto y, desde luego, no es enseñanza virtual. El problema es que muchos docentes han tenido que adaptarse de manera precipitada a un modelo de enseñanza virtual para el que no estaban preparados (vicerrectora de Estudiantes).

A continuación, se presentan los datos relativos al uso de herramientas digitales por parte de los docentes antes del confinamiento y durante el mismo (tabla 4). Los recursos más utilizados por 'todos' los docentes antes de la suspensión de la enseñanza presencial eran, para el 10,8\% de los estudiantes, los del campus virtual -foro, chat, tareas, etc.-. Sin embargo, durante la pandemia, el porcentaje de estudiantes que afirma que sus docentes utilizan este tipo de herramientas asciende al 23,7\%. Igualmente aumenta el porcentaje de estudiantes que indican que 'todos' sus profesores elaboran videotutoriales $(2,7 \%)$ o utilizan videotutoriales elaborados por otros $(1,3 \%)$. Más significativo es aún el dato que revela que 'la mayoría' de los profesores han realizado sus propios videos $(11,7 \%)$ o han recurrido a los de otros $(4,4 \%)$ para sus sesiones de ED. Es, sin embargo, el uso de videotutoriales por parte de 'algunos' docentes el que experimenta una mayor subida al pasar de un 13,0\% a un $66,8 \%$, en el caso de docentes que producen sus propios videotutoriales; o de un $20,8 \%$ a un 30,5\%, en el de los que recurren a videos de otros. En coherencia con estos datos, también se produce un aumento del uso de la videoconferencia por 'algunos' profesores para interactuar con los estudiantes que pasa de un 4,2\% al $66,4 \%$. En general, se mantienen prácticamente inalterados los porcentajes de aquellos que señalan que 'todos' ( $1,1 \%$ antes y durante el confinamiento), 'la mayoría' ( $1,3 \%$ antes y $3,5 \%$ durante) o 'algunos' (17.0\% antes y $18,4 \%$ durante) hacen un uso residual de las redes sociales. Lo mismo puede decirse de los que acuden al blog del docente como una herramienta más en su práctica de enseñanza online.

Tabla 4. Uso de herramientas digitales docentes antes y durante el confinamiento

\begin{tabular}{|l|c|c|c|c|c|c|c|c|c|c|c|c|c|}
\hline & $\begin{array}{l}\text { Videotutoriales } \\
\text { elaborados por } \\
\text { el docente }\end{array}$ & \multicolumn{2}{|c|}{$\begin{array}{l}\text { Videotutoriales } \\
\text { elaborados por } \\
\text { otros docentes }\end{array}$} & \multicolumn{2}{|c|}{ Blog docente } & \multicolumn{2}{|c|}{$\begin{array}{c}\text { Videoconferencias } \\
\text { para interactuar } \\
\text { con los estudiantes }\end{array}$} & \multicolumn{2}{|c|}{$\begin{array}{c}\text { Herramientas } \\
\text { del Campus } \\
\text { Virtual }\end{array}$} & \multicolumn{2}{|c|}{ Redes Sociales } \\
\hline & Antes & Durante & Antes & Durante & Antes & Durante & Antes & Durante & Antes & Durante & Antes & Durante \\
\hline Todos & 0.4 & 2.7 & 0.2 & 1.3 & 2.0 & 1.5 & 0.2 & 3.3 & 10.8 & 23.7 & 1.1 & 1.1 \\
\hline La mayoría & 1.6 & 11.7 & 0.7 & 4.4 & 4.7 & 5.1 & 0.9 & 16.6 & 32.1 & 35.2 & 1.3 & 3.5 \\
\hline Algunos & 13.0 & 66.8 & 20.8 & 36.5 & 23.4 & 22.6 & 4.2 & 66.4 & 51.1 & 36.3 & 17.0 & 18.4 \\
\hline Ninguno & 85.0 & 18.8 & 78.3 & 57.8 & 69.9 & 70.8 & 94.7 & 13.7 & 6.0 & 4.7 & 80.7 & 77.0 \\
\hline TOTAL & $100 \%$ & $100 \%$ & $100 \%$ & $100 \%$ & $100 \%$ & $100 \%$ & $100 \%$ & $100 \%$ & $100 \%$ & $100 \%$ & $100 \%$ & $100 \%$ \\
\hline
\end{tabular}

Fuente: Elaboración propia 


\section{Valoración que hacen los estudiantes de la enseñanza online recibida}

El tercer objetivo de la investigación tenía como finalidad conocer la valoración de los estudiantes acerca de la ED impartida por sus docentes. Con tal propósito se les preguntó por la dedicación que para ellos conllevaba esta modalidad y si se traducía en un mayor rendimiento educativo. Además, se les pedía una valoración sobre la importancia de usar las tecnologías educativas digitales en la universidad y si la educación online podría sustituir a la enseñanza presencial. Por último, se indagaba en la comunicación y el diálogo establecida entre docentes y estudiantes para abordar no solo aspectos académicos vinculados con la metodología, la evaluación, los contenidos, etc., sino aquellos otros con un cariz más personal referidos a la adaptación de los docentes a las singulares circunstancias en las que los estudiantes estaban recibiendo la ED.

Los datos (tabla 5) revelan que para más del $75 \%$ la ED ha exigido mayor dedicación en la preparación de las asignaturas que el destinado en el formato presencial. A pesar del incremento en el tiempo de estudio un porcentaje similar $(77,3 \%)$ no percibe proporcionalidad en el rendimiento académico. Esta inversa relación entre dedicación y percepción del rendimiento puede ser uno de los factores que contribuyen a explicar su 'desacuerdo' (25,5\%) o 'total desacuerdo' (41,2\%) con el hecho de que las clases online puedan sustituir a las presenciales. Sin embargo, un 85,2\% está de 'acuerdo' o 'totalmente de acuerdo' en que la universidad no puede prescindir del uso educativo de las TIC. Existe un alto consenso en señalar que los docentes no se han adaptado a las excepcionales circunstancias que vivían los alumnos en su ámbito personal $(65,1 \%)$ o académico $(68,1 \%)$.

Tabla 5. Valoración de los estudiantes de la enseñanza recibida

\begin{tabular}{|l|c|c|c|c|c|}
\hline & $\begin{array}{c}\text { Totalmente } \\
\text { en } \\
\text { desacuerdo }\end{array}$ & $\begin{array}{c}\text { En } \\
\text { desacuerdo }\end{array}$ & $\begin{array}{c}\text { Ni de } \\
\text { acuerdo } \\
\text { ni en } \\
\text { desacuerdo }\end{array}$ & $\begin{array}{c}\text { De } \\
\text { acuerdo }\end{array}$ & $\begin{array}{c}\text { Totalmente } \\
\text { de acuerdo }\end{array}$ \\
\hline $\begin{array}{l}\text { La enseñanza virtual } \\
\text { exige mayor dedicación } \\
\text { a la preparación de las } \\
\text { asignaturas. }\end{array}$ & $4,9 \%$ & $8,6 \%$ & $11,3 \%$ & $20,6 \%$ & $54,6 \%$ \\
\hline $\begin{array}{l}\text { Estoy aprendiendo más con } \\
\text { esta modalidad de enseñanza. }\end{array}$ & $51,6 \%$ & $25,7 \%$ & $16,6 \%$ & $4,6 \%$ & $1,5 \%$ \\
\hline $\begin{array}{l}\text { Las clases virtuales pueden } \\
\text { sustituir a las presenciales. }\end{array}$ & $41,2 \%$ & $25,5 \%$ & $14,1 \%$ & $13 \%$ & $6,2 \%$ \\
\hline $\begin{array}{l}\text { El uso educativo de las TIC } \\
\text { es imprescindible en la } \\
\text { Universidad. }\end{array}$ & $1,8 \%$ & $3,1 \%$ & $9,9 \%$ & $39,4 \%$ & $45,8 \%$ \\
\hline
\end{tabular}




\begin{tabular}{|l|c|c|c|c|c|}
\hline & $\begin{array}{c}\text { Totalmente } \\
\text { en } \\
\text { desacuerdo }\end{array}$ & $\begin{array}{c}\text { En } \\
\text { desacuerdo }\end{array}$ & $\begin{array}{c}\text { Ni de } \\
\text { acuerdo } \\
\text { ni en } \\
\text { desacuerdo }\end{array}$ & $\begin{array}{c}\text { De } \\
\text { acuerdo }\end{array}$ & $\begin{array}{c}\text { Totalmente } \\
\text { de acuerdo }\end{array}$ \\
\hline $\begin{array}{l}\text { Los docentes han adaptado la } \\
\text { enseñanza virtual a nuestras } \\
\text { circunstancias personales. }\end{array}$ & $27 \%$ & $38,1 \%$ & $21,2 \%$ & $12,2 \%$ & $1,5 \%$ \\
\hline $\begin{array}{l}\text { Los docentes han } \\
\text { consensuado con los } \\
\text { estudiantes las decisiones } \\
\text { académicas. }\end{array}$ & $39.1 \%$ & $29 \%$ & $18 \%$ & $10,8 \%$ & $3,1 \%$ \\
\hline
\end{tabular}

Fuente: Elaboración propia

La valoración de los estudiantes sobre la enseñanza online recibida coincide con los resultados de las entrevistas a miembros del equipo de gobierno de la UEx.

Hemos recibido los resultados de un cuestionario elaborado por el Consejo de Estudiantes sobre el nivel de satisfacción con las enseñanzas virtuales donde se pone de manifiesto que algunos docentes no se han preocupado por conocer si las circunstancias personales de los estudiantes les permitían seguir las clases online. También señalan que algunos profesores les han sobrecargado de tareas y otros, los menos, no les han proporcionado orientaciones sobre la forma en que se iba a impartir su asignatura. Todo ello ha generado en algún caso un cierto estrés y el sentimiento de que sus resultados académicos podían empeorar (vicerrectora de Estudiantes)

\section{DISCUSIÓN Y CONCLUSIONES}

A tenor de los resultados que se derivan del estudio, el alumnado de la UEx podría enmarcarse en el perfil de «estudiantes típicos», esto es, personas jóvenes, solteras, sin hijos, sin empleo ni búsqueda de éste y, por tanto, dependientes de los ingresos de una unidad familiar en la que, de forma mayoritaria, los progenitores poseen estudios superiores. En este sentido, los hallazgos indican que las circunstancias personales no tienen incidencia significativa en la equidad. No puede decirse lo mismo respecto al nivel formativo de los padres, dado que son los hijos e hijas de las familias con un nivel formativo alto o medio-alto los que cuentan con un ordenador de uso exclusivo y una conectividad propia y de alta velocidad para un correcto seguimiento de la enseñanza virtual. Coincidiendo con los resultados de un reciente estudio de Cabrera et al. (2020) referidos a la enseñanza primaria, esta investigación constata que el nivel formativo de las familias, aunque no de manera exclusiva, actúa como gradiente de la desigualdad. Así, el riesgo de interrupción y abandono educativo puede verse agravado por la brecha de uso (UNESCO, 2020).

Los hallazgos relacionados con el segundo objetivo de la investigación, conocer el modelo de enseñanza a distancia que han recibido los estudiantes, confirman el 
predominio de las presentaciones multimedia como recurso didáctico de los docentes y un uso residual de los blogs y las redes sociales, a pesar de la actitud positiva que muestran los estudiantes en el uso de redes sociales (Gómez-Aguilar et al., 2012). En línea con otros estudios (Mercader, 2019; Mercader y Gairín, 2017; Prendes, 2010; Venegas-Ramos et al., 2020), se constata que el uso de las tecnologías digitales en la ED ha consistido, esencialmente, en trasladar los contenidos impartidos en las clases presenciales a plataformas de aprendizaje virtual. Este resultado viene a corroborar que los docentes hacen uso de aquellas tecnologías que son coherentes con sus prácticas docentes (Marcelo-García et al., 2015).

Por otra parte, los docentes han optado mayoritariamente por un modelo asíncrono acompañado de una interacción docente-discente basada en la comunicación a través de correo electrónico, foros o chat.Y este es un aspecto relevante si se tienen en cuenta los riesgos de abandono asociados a una desvinculación entre estudiantes y profesores (Alzás y Casas, 2015).

La valoración que hacen los estudiantes con respecto a la ED recibida durante el confinamiento se fundamenta principalmente en la inversa relación entre dedicación al estudio y rendimiento percibido y en la ausencia de concertación entre docentes y alumnado que se concreta en la falta de adaptación de los primeros a las circunstancias personales de los segundos. De estos resultados se infiere que la comunicación es un factor determinante para evitar el efecto de aislamiento que suele producirse en los modelos de enseñanza a distancia; efecto agudizado, además, por el impacto psicológico que la reclusión forzosa por la pandemia podía provocar. Los estudiantes también se han visto obligados a adaptarse a un modelo formativo cuyos contenidos estaban diseñados para la presencialidad y que les exigía una mejor gestión del tiempo y, por tanto, más disciplina y organización. De hecho, autores como Giesbers et al. (2013) y Moallen (2015) han evidenciado la preferencia de los estudiantes por modelos de aprendizaje mixtos que combinen lo síncrono y lo asíncrono.

Lo que subyace, en definitiva, a tenor de los resultados de la investigación, es la raigambre de un paradigma tradicional asentado en clases magistrales que debe dar paso a otros modelos más colaborativos y centrados en el estudiante. El cierre de las aulas presenciales representa una oportunidad para este cambio una vez superada la barrera tecnológica que dificultaba el uso de tecnologías digitales por parte de los docentes. La transición hacia una digitalización integral solo será posible si va acompañada de un marco estratégico (García-Peñalvo, 2020) que produzca una transformación en el modelo institucional de la universidad junto con sus principales protagonistas, docentes y estudiantes. Además, la importante inversión en recursos humanos y económicos realizada por las universidades presenciales debe ser capitalizada y orientada a ampliar su oferta educativa convirtiendo algunos títulos presenciales en títulos en la modalidad blended-learning o completamente online. Muchos han percibido las posibilidades de ampliar un mercado, el de las universidades virtuales, que ofrece ya una gran diversidad de titulaciones. Las 
universidades presenciales, que venían compitiendo con estas otras y que continuarán haciéndolo con más intensidad si cabe, deben adoptar elementos diferenciadores que aporten valor a sus titulaciones.

Futuras líneas de investigación podrían ampliar el alcance del estudio y abordar, desde la perspectiva del profesorado, el modelo de enseñanza a distancia que han implementado durante la suspensión de las clases presenciales e identificar las dificultades que han enfrentado unos y las buenas prácticas de otros. El empeño de este artículo no es otro que presentar mediante evidencias empíricas un diagnóstico con luces y sombras sobre la enseñanza a distancia impartida durante el confinamiento que sirva a las universidades para afrontar los retos que sigue deparando el COVID-19.

\section{REFERENCIAS}

Alva de la Selva, A. R. (2014). Los nuevos rostros de la desigualdad en el siglo XXI: La brecha digital. Revista Mexicana de Ciencias Políticas y Sociales, 3(223), 265-286. https://doi.org/10.1016/S01851918(15)72138-O

Alzás, T., y Casas, L. (2015). Relato autobiográfico del abandono educativo. Una visión integrada desde metodologías mixtas. Investigación Cualitativa en Ciencias Sociales, 3, 108-113. https://bit. ly/3l51Ez7

Ariño, A., Martínez, M., Llopis, R., Pons, E., y Prades, A. (2019). Via Universitària: Accés, condicions d'aprenentatge, expectatives $i$ retorns dels estudis universitaris (2017-2019). Valencia: Xarxa Vives. https://bit.ly/3eYN8Wd

Baker, D. (2011). Designing and orchestrating online discussions. MERLOT Journal of Online Learning and Teaching, 7(3), 401411. https://bit.ly/3goHYTN

Banco Mundial (2020). The COVID-19 pandemic shocks to Education and Policy Responses. https://bit.ly/3gmJtSc

Beltrán Llavador, J., Venegas, M., VillarAguilés, A., Andrés-Cabello, S., JareñoRuiz, D., y de Gracia-Soriano, P. (2020). Educar en época de confinamiento: la tarea de renovar un mundo común. Revista de Sociología de la Educación-RASE, 13(2),
Especial, COVID-19, 92-104. https://doi. org/10.7203/RASE.13.2.17187

Brodie, R. J., Ilic, A., Juric, B., y Hollebeek, L. (2013). Consumer engagement in a virtual brand community: An exploratory analysis. Journal of Business Research, 66, 105-114. https://doi.org/10.1016/j. jbusres.2011.07.029

Cabrera, L. (2020). Efectos del coronavirus en el sistema de enseñanza: aumenta la desigualdad de oportunidades educativas en España. Revista de Sociología de la Educación-RASE, 13(2), Especial, COVID-19, 114-139. https://doi. org/10.7203/RASE.13.2.17125

Cabrera, L., Pérez, C. N., y Santana, F. (2020). ¿Se incrementa la Desigualdad de Oportunidades Educativas en la Enseñanza Primaria con el Cierre Escolar por el Coronavirus? International Journal of Sociology of Education, Special Issue: COVID-19 Crisis and Socioeducative Inequalities and Strategies to Overcome them, 27-52. https://doi.org/10.17583/ rise.2020.5613

Castaño-Muñoz, J., Duart, J., y Vinuesa, T. (2014). The internet in face-to-face higher education: Can interactive learning improve academic achievement? British Journal of Educational Technology, 
45(1), 149-159. https://doi.org/10.1111/ bjet.12007

Chakraborty, M., y Nafukho, F. M. (2015). Strategies for virtual learning environments: Focusing on teaching presence and teaching immediacy. Internet Learning, 4(1). https://bit. ly/2YZc36r. https://doi.org/10.18278/ il.4.1.1

Duncan, K., Kenworthy, A., y McNamara, R. (2012). The effect of synchronous and asynchronous participation on students' performance in online accounting courses. Accounting Education: An International Journal, 21, 431-449. https://doi.org/10. 1080/09639284.2012.673387

Falloon, G. (2011). Making the connection: Moore's theory of transactional distance and its relevance to the use of a virtual classroom in postgraduate online teacher education. Journal of Research on Technology, 43, 187-209. https://doi.org /10.1080/15391523.2011.10782569

Fariña-Vargas, E., González-González, C., y Area-Moreira, M. (2013). ¿Qué uso hacen de las aulas virtuales los docentes universitarios? Revista de Educación a Distancia, 35. https://bit.ly/2C2NMng

Fernández Enguita, M. (2016). La Educación en la encrucijada. Fundación Santillana. https://bit.ly/34hPqxc

Francescucci, A., y Rohani, L. (2018). Exclusively Synchronous Online (VIRI) Learning: The Impact on Student Performance and Engagement Outcomes. Journal of Marketing Education, 41(1), 60-69. https://doi. org/10.1177/0273475318818864

García-Peñalvo, F. J. (2020). Modelo de referencia para la enseñanza no presencial en universidades presenciales. Campus Virtuales, 9(1), 41-56. https:// bit.ly/3gv6oer

Giesbers, B., Rienties, B., Tempelaar, D., y Gijselaers, W. (2013). A dynamic analysis of the interplay between asynchronous and synchronous communication in online learning: The impact of motivation. Journal of Computer Assisted Learning, 30, 30-50. https://doi.org/10.1111/ jcal.12020

Goldman, Z. (2011). Balancing quality and workload in asynchronous online discussions: A win-win aroach for students and instructors. MERLOT Journal of Online Learning and Teaching, 7(2), 313323. https://bit.ly/2ZJgLEK

Gómez-Aguilar, M., Roses-Campos, S., y Farias-Batlle, P. (2012). The academic use of social networks among university students. Comunicar, 38, 131-138. https://doi.org/10.3916/C38-2012-03-04

Harari, Y. N. (2016). Homo Deus. Breve historia del mañana. Debate. https://doi. org/10.17104/9783406704024

Hrastinski, S. (2008). Asynchronous and synchronous e-learning. Educause Quarterly, 31(4), 51-55. https://bit. ly/2ByYbqP

Hodges, Ch., Moore, S., Lockee, B., Trust, T., y Bond, A. (2020). The Difference Between Emergency Remote Teaching and Online Learning. Educause Review. https://bit.ly/3dZlh6U

Hogg, N., y Lomicky, C. (2012). Connectivism in postsecondary online courses: An exploratory Factor Analysis. The Quarterly Review of Distance Education, 13(2), 95-114. https://bit.ly/2Ooo3yd

Llorente, M. C., y Cabero, J. (2009). La formación semipresencial a través de redes telemáticas (blended learning). Mataró: Da Vinci.

Maor, D., y Currie, J. K (2017). The use of technology in postgraduate supervision pedagogy in two Australian universities. International Journal of Educational Technology in Higher Education, 14(1), 1-15. https://doi.org/10.1186/s41239017-0046-1

Marcelo-García, C., Yot-Domínguez, C., y Mayor-Ruiz, C. (2015). University teaching with digital technologies. 
Comunicar, 45, 117-124. https://doi. org/10.3916/C45-2015-12

Mercader, C. (2019). Las resistencias del profesorado universitario a la utilización de las tecnologías digitales. Aula Abierta, 48(2), 167-174. https://doi.org/10.17811/ rifie.48.2.2019.167-174

Mercader, C., y Gairín, J. (2017). ¿Cómo utiliza el profesorado universitario las tecnologías digitales en sus aulas? REDURevista de Docencia Universitaria, 15(2), 257-273. https://doi.org/10.4995/ redu. 2017.7635

Moallem, M. (2015). The impact of synchronous and asynchronous communication tools on learner selfregulation, social presence, immediacy, intimacy, and satisfaction in collaborative online learning. The Online Journal of Distance Education and e-learning, 3(3), 55-77. https://bit. $\underline{\mathrm{ly} / 3 \mathrm{aK} 571 \mathrm{c}}$

Moore, M. G., y Kearsley, G. (Eds.) (2012). Distance education: A systems view of online learning. Belmont: Wadsworth.

OCDE (2020). A helping hand: Education responding to the coronavirus pandemic. https://bit.ly/2VM5No1

OEI (2020). Efectos de la crisis del coronavirus sobre la educación. https:// bit.ly/2YWKVoB

Prendes, M. P. (2010). Competencias TIC para la docencia en la Universidad pública española: indicadores y propuestas para la definición de buenas prácticas. Informe del Proyecto EA-2009-0133 de la Secretaría de Estado y Universidades e Investigación. https://bit.ly/3f6JRV2

Ragusa, A. T. (2017). Technologically mediated communication: student expectations and experiences in a FOMO society. International Journal of Educational Technology in Higher Education, 14, 39. https://doi. org/10.1186/s41239-017-0077-7

REACU (2020). Aplicación del documento REACU "orientaciones para la elaboración y evaluación de títulos de grado y máster en enseñanza no presencial y semipresencial". https://bit. ly/3eoYOgt

Sevillano-García, M., Quicios-García, M., y González-García, J. (2016). The ubiquitous possibilities of the laptop: Spanish university students' perceptions. Comunicar, 46, 87-95. https://doi. org/10.3916/C46-2016-09

Strang, K. (2013). Cooperative learning in graduate student projects: Comparing synchronous versus asynchronous collaboration. Journal of Interactive Learning Research, 24, 447-464. https:// bit.ly $/ 2 Z_{3}$ Cisq

UNESCO (2020). COVID-19 y educación superior: De los efectos inmediatos al día después. https://bit.ly/2ZyhQyW

UNICEF (2020). La educación frente al COVID-19. Propuestas para impulsar el derecho a la educación durante la emergencia. https://bit.ly/2BEUpvN

Venegas-Ramos, L., Luzardo Martínez, H.J., y Pereira Santana, A. (2020). Conocimiento, formación y uso de herramientas TIC aplicadas a la Educación Superior por el profesorado de la Universidad Miguel de Cervantes. Edutec. Revista Electrónica de Tecnología Educativa, 71, 35-52. https:// doi.org/10.21556/edutec.2020.71.1405

Vlachopoulos, D., y Makri, A. (2019). Online communication and interaction in distance higher education: A framework study of good practice. International Review of Education, 65, 605-632. https://doi.org/10.1007/s11159-01909792-3

Watts, L. (2016). Synchronous and asynchronous communication in distance learning: A review of the literature. The Quarterly Review of Distance Education, 17(1), 23-32. https://bit.ly/2YlBmz2

Yang, Z., y Liu, Q. (2007). Research and development of Web-based virtual online classroom. Computers \& Education, 
48, 171-184. https://doi.org/10.1016/j. compedu.2004.12.007

Zubillaga, A., y Gortazar, L. (2020). COVID-19 y educación: Problemas, respuestas y escenarios. Fundación COTEC para la innovación. https://bit. ly/2AtszSH

\section{PERFIL ACADÉMICO Y PROFESIONAL DE LOS AUTORES}

Eva Pérez-Lopez. Profesora sustituta en el Departamento de Dirección de Empresa y Sociología (Área De Sociología) de la Universidad de Extremadura; profesora-tutora en los grados de Sociología y Ciencia Política de la UNED. Líneas de investigación: global education policy; políticas públicas relacionadas con las TIC; comunicación política.

E-mail: evaperez@unex.es

Dirección

Centro Universitario de Plasencia

C/Virgen del Puerto, 2

Plasencia (España)

orcid.org/0000-0002-6580-7038

Alfonso Vázquez Atochero, profesor ayudante en el Departamento de Ciencias de la Educación (área Didáctica y Organización Escolar) de la Universidad de Extremadura. Líneas de investigación: humanidades digitales; relación entre el ser humano y el ciberespacio.

E-mail: alfonso@unex.es

Dirección:

Facultad de Formación del Profesorado

Avda. de la Universidad $\mathrm{s} / \mathrm{n}$

Cáceres (España)

orcid.org/0000-0002-1657-8275

Santiago Cambero Rivero, profesor asociado en el Departamento de Dirección de Empresas y Sociología (área de Sociología) de la Universidad de Extremadura. Líneas de investigación: aprendizaje intergeneracional, envejecimiento activo, cibervoluntariado.

E-mail: scamriv@unex.es

Dirección:

Avda. de Elvas s/n

Badajoz (España)

orcid.org/0000-0001-8371-8349 
Fecha de recepción del artículo: 06/07/2020

Fecha de aceptación del artículo: 25/08/2020

Fecha de aprobación para maquetación: 02/09/2020 